\title{
The depletion of MARVELD1 leads to placenta accreta via integrin
}

\section{B4-dependent trophoblast cell invasion}

3

4 Yue Chen ${ }^{\dagger}$, Hui Zhang ${ }^{\dagger}$, Fang Han, Lei Yue, Chunxiao Qiao, Yao Zhang, Peng Dou, 5 Weizhe Liu, Yu Li

6

7 School of life science and technology, Harbin Institute of Technology, Harbin, China 8

$9+$ These authors contributed equally to this work

10 Author for correspondence (liyugene@hit.edu.cn)

11

12

13

KEY WORDS: MARVELD1; placenta accrete; integrin $\beta 4$; cell adhesion; trophoblast 14 cell; cell invasion 


\section{Abstract}

17

18 The mammalian placenta is a remarkable organ. It serves as the interface between

19 the mother and the fetus. Proper invasion of trophoblast cells into the maternal

20 decidua is required for a successful pregnancy. Previous studies have found that the

21 adhesion molecule integrin $\beta 4$ plays important roles during trophoblast cell invasion.

22 Here, we found that the overall birth rate of the MARVELD1 knockout mouse is much

23 lower than that of the wild-type mouse $(P<0.001)$. In E18.5 MARVELD1 knockout

24 mice, we observed an over-invasion of trophoblast cells, and indeed, the pregnant

25 mice had a partial placenta accreta phenotype. The HTR8/SVneo cell line was used as

26 an in vitro model to elucidate the underlying mechanisms of MARVELD1-mediated

27 trophoblast invasion. We detected a diminished expression of integrin $\beta 4$ upon the

28 downregulation of MARVELD1 and enhanced migration and invasive abilities of

29 trophoblast cells both in vivo and in vitro. The integrin $\beta 4$ rescue assay also

30 supported the results. In conclusion, this study found that MARVELD1 mediated the

31 invasion of trophoblast cells via regulating the expression of integrin $\beta 4$. 


\section{Introduction}

The mammalian placenta is a remarkable organ. The robust growth of the fetus is dependent on it. It forms the interface between the mother and fetus and is needed for gas and nutrient exchange as well as waste disposal. In addition, the placenta acts as a barrier against the maternal immune system by producing hormones and growth factors(Rossant and Cross, 2001; Watson and Cross, 2005). In humans and mice, the mature placenta is composed of three major layers: the outer layer is called the maternal decidua, which contains decidual cells of the uterus as well as the maternal vasculature that mediates blood exchange with the implantation site. The middle layer acts as a "junctional region", which is important for the attachment of the placenta to the uterus; it contains trophoblast cells that invade the maternal decidua. The inner layer is called the labyrinth, which is composed of an abundance of branched villi for efficient gas and nutrient exchange(Rossant and Cross, 2001). Although the detailed architecture of the placenta in humans and mice are somewhat different, their overall structures and the molecular mechanisms are quite similar(Rossant and Cross, 2001). Thus, the mouse is an ideal model for studying placental development.

During placenta development in humans, the cytotrophoblast cells from anchor villi differentiate into extravillous trophoblasts (EVTs), lose its proliferative phenotype, and gain an invasive phenotype(Irving et al., 1995), forming three major subtypes: villous cytotrophoblasts (CTBs), syncytiotrophoblasts and EVTs(Fitzgerald et al., 2008; Gude et al., 2004; Lash, 2015). Similar to cancer progression, which involve cellular movement into foreign tissue, trophoblast invasion into the maternal decidua is tightly controlled by many adhesion molecules, including integrins(Damsky et al., 1992; Damsky et al., 1994), matrix metalloproteinases (MMPs) and metalloproteinases inhibitors (TIMPs) (Anacker et al., 2011; Bischof et al., 2000; Godbole et al., 2011). In particular, cytotrophoblast cell invasiveness is associated 
with different cell surface molecules, which include integrins. Integrins are a family of heterodimeric transmembrane receptors that are formed by two different chains, the $\alpha$ (alpha) and $\beta$ (beta) subunits, and are expressed on the basal cell surface; it mediates cell adhesion to extracellular matrix (ECM) though the amino acid sequence Arginine-Glycine-Aspartic acid (RGD) (Ruoslahti, 1991; Ruoslahti, 1996). Integrins play important roles in cell adhesion and migration. During the invasion process, the expression of integrin $\alpha 1 \beta 1$ and $\alpha 5 \beta 1$ are upregulated whereas integrin a6ß4 is downregulated (Damsky et al., 1994; Maltepe and Fisher, 2015).

Cellular invasion during placenta development, especially cytotrophoblast differentiation, is a complex process that is tightly regulated. Proper invasion of trophoblast cells into the maternal decidua is required for successful pregnancy(Huppertz, 2008). Disruption of this tightly controlled process can lead to placental deficiencies, resulting in pregnancy complications(Lash, 2015) such as early miscarriage(Hustin et al., 1990), late miscarriage(Ball et al., 2006), preeclampsia(Pijnenborg et al., 1991), fetal growth restriction(Khong et al., 1986), preterm birth(Kim et al., 2003), and placenta accreta(Khong and Robertson, 1987). Placenta accreta is a disorder of human placentation characterized by the abnormal attachment or invasion of placental tissue into the underlying uterine musculature (Dashraath and Lin, 2016; Jauniaux et al., 2016; Luke et al., 1966). Despite the importance of cytotrophoblast cell invasion in placenta development, very little is known about the factors that control this process in vivo.

Nuclear factor MARVELD1 (MARVEL domain-containing 1) is a novel tumor suppressor candidate, which is widely expressed in human normal tissues but is down regulated in multiple carcinomas by promoter methylation (Wang et al., 2009). Previous studies have found that mouse MARVELD1 is a nuclear protein that inhibits cell migration (Zeng et al., 2011). Notably, in non-small cell lung cancer (NSCLC), MARVELD1 regulates the balance of integrin $\beta 1 / \beta 4$, integrin $\beta 1 / \beta 4$-mediated cell surface ultrastructure, and EMT (Yao et al., 2015). Here, we addressed the role of 
90 MARVELD1 in mouse placenta development. We demonstrate that the knockout of

91 MARVELD1 in mouse placenta induced the downregulation of integrin $\beta 4$ and further

92 suppressed integrin $\beta 4$-mediated cell adhesion; therefore, this novel nuclear factor

93 plays an essential role in the regulation of integrin $\beta 4$ and associated functions.

94 These molecular mechanism triggers the over-invasion of trophoblast cells, which

95 leads to placenta accreta and a difficult birthing process in MARVELD1/- mice. This

96 study accelerated our efforts in understanding the critical role of the placenta in

97 successful pregnancies.

98 
Materials and methods

100

101 Cell lines and reagents

102 Human cell lines NIH/3T3, NCl-H460, MDA-MB-231, NCl-H28, NCl-H2452, 103 HTR-8/SVneo, IRR-MRC-5, and WI38 were purchased from the American Type Culture 104 Collection (ATCC, Manassas, VA). Stable cell lines NIH/3T3-MARVELD1-V5 and $105 \mathrm{NIH} / 3 \mathrm{T3}-\mathrm{PC}$ cells were maintained by our lab. Antibodies against MARVELD1 106 (ab91640), Cytokeratin18 (ab668), and integrin $\beta 4$ (ab182120) were purchased from 107 Abcam (Cambridge, MA). Antibodies against V5 (\#13202) were purchased from Cell 108 Signaling Technology. Antibody against GAPDH (TA-08) was purchased from ZSGB-BIO 109 (ZSGB-BIO, Beijing, China). Laminin (L4544) was purchased from Sigma, TGF- $\beta 1$ 110 (240-B) was purchased from R\&D, Lipofectamine ${ }^{\circledR} 2000$ transfection reagent 111 (Cat\#11668019) and cell tracker (Cat\#C34552) were purchased from Invitrogen, 112 TRIzol reagent (Cat\#11667165001) and FastStart Universal SYBR Green Master (ROX) 113 (Cat\#04913850001) were purchased from Roche, the PrimeScript ${ }^{\mathrm{TM}} \mathrm{RT}$ reagent Kit 114 with gDNA Eraser (Cat\#RR047Q) was purchased from Takara, and the Dual-Luciferase 115 reporter assay system (Catalog no. E1910) was purchased from Promega.

\section{MARVELD1 knockout mice}

118 All animal experiments were performed in strict accordance with the 119 recommendations in the Guide for the Care and Use of Laboratory Animals from the 120 Harbin Institute of Technology. The MARVELD1 knockout mice were generated by 121 Biocytogen (Beijing, CHINA) by injecting conditional MARVELD1 knockout murine ES 122 cells (conditional Cre-loxP target vector cassette) into blastocysts with a C57BL/6 123 background (C57BL/6J-Tyrc-2J) to obtain chimeric mice. MARVELD1 heterozygous 124 animals were backcrossed to wild-type C57BL/6 mice to obtain conditional 125 MARVELD1 knockout mice without the albino phenotype (Tyrc-2J). Then, the 
127 MARVELD1 knockout mice. Mice were genotyped by PCR using primers that detected

128 wild-type and knockout sequences. Primers sequences are available in the Table S1.

129

\section{Cell culture and RNA interference}

131 HTR-8/SVneo cells were derived by transfecting the cells that grew from chorionic 132 villi explants of human first-trimester placenta with the gene encoding simian virus 13340 large $T$ antigen. These transfected trophoblasts were used for the study of 134 trophoblast biology and placental function. Cells were cultured following the 135 protocol from ATCC (Gibco, NY). Cells were cultured in RPMI-1640 medium with 5\% 136 fetal bovine serum at $37^{\circ} \mathrm{C}$ in an incubator with $5 \% \mathrm{CO}_{2}$.

137 For RNA interference, MARVELD1 siRNAs and scrambled siRNAs were designed by 138 the GenePharma Company. Cells were transfected with Lipofectamine ${ }^{\circledR} 2000$ 139 Transfection Reagent following the manufacturers' protocol. The knockdown 140 efficiency of the siRNAs was determined by real-time PCR.

\section{Reverse transcription and real-time PCR}

143 Total RNA was extracted and purified using the TRIzol reagent. The concentration of 144 each RNA sample was determined by using the NanoDrop 2000 spectrophotometer 145 (Thermo Fisher Scientific Inc.). One $\mu \mathrm{g}$ of total RNA was used as a template for 146 reverse transcription into $\mathrm{cDNA}$ using the PrimeScript ${ }^{\mathrm{TM}} \mathrm{RT}$ reagent Kit with gDNA 147 Eraser according to the manufacturer's instructions. Primers are available in the 148 Table S1.

149 Quantitative real-time PCR was performed with FastStart Universal SYBR Green 150 Master (ROX) according to the manufacturer's instructions using the ViiTM 7 151 Real-time PCR system (Applied Biosystems, Foster City, CA, USA).

152

153 Western blotting

154 Western blotting was performed as described previously. Briefly, total protein was 
155

156

157

158

159

160

161

162

163

164

165

166

167

168

169

170

171

172

173

174

175

176

177

178

179

180

181

182

separated on a precast $12 \%$ polyacrylamide gel and blotted with antibodies for integrin $\beta 4$ (diluted 1:1000), integrin $\beta 1$ (diluted 1:1000) and GAPDH (diluted 1:20000). Data were analyzed and quantified by using the Odyssey infrared imaging system application software v3.0.

\section{Histological analysis}

For placental tissue, $5 \mu \mathrm{m}$ sections were used. Sections were collected and fixed in $4 \%$ paraformaldehyde, dehydrated, embedded in paraffin and sectioned. Histological sections were stained with hematoxylin and eosin (H\&E) or used for other analyses such as immunohistochemistry (IHC) and immunofluorescence (IF) staining assays.

\section{IHC and IF}

Sections were washed three times in PBST (PBS with 0.1\% Triton X-100), blocked with $10 \%$ normal serum/PBST from the same species as the secondary antibody, and incubated overnight at $4{ }^{\circ} \mathrm{C}$ with primary antibodies: Mouse anti-Cytokeratin 18(1:500), Rabbit anti-integrin $\beta 4$ (1:250), and MARVELD1 (1:100). The primary antibodies were removed, and the sections were washed three times with PBST for 5 minutes each.

For IHC, 100-400 $\mu \mathrm{l}$ of biotinylated secondary antibodies, diluted in TBST per the manufacturer's recommendation, was added to each section. Then, the antibody staining was developed using established procedures.

For IF, the antibodies were applied in the same manner as for cell staining. The secondary antibodies were from Invitrogen. Stained sections were visualized using a Zeiss AXIO Zoom.V16 Stereo Zoom Microscope.

For cell staining, cells were fixed in $4 \%$ paraformaldehyde for 15 minutes on ice, blocked with $5 \%$ normal serum from the same species as the secondary antibody, and stained using established procedures. Trophoblast cells were detected using anti Cytokeratin18 (1:500) and anti-integrin $\beta 4$ (1:250) antibodies. Stained cells were 
183 visualized using a Zeiss LSM 510 Meta Confocal Microscope.

184

185 Transwell cell migration and invasion assays

186 A total of $1.5 \times 10^{4}$ cells were seeded onto the upper chamber of 24-well Transwell

187 plates. Medium containing $10 \%$ FBS was placed in the lower chamber and served as a

188 chemoattractant. Cells were allowed to migrate for 18 hours at $37^{\circ} \mathrm{C}$, the cells on the

189 upper surface of the filter were removed by gently wiping with a cotton swab. The

190 cells that had migrated to the Transwell were fixed and stained with crystal violet.

191 The migrated cells were visualized by a microscope.

192 For the invasion assay, 1\% Matrigel was added to the upper chamber before cell

193 migration, and cells were allowed to migrate for 24 hours at $37^{\circ} \mathrm{C}$.

194

195 Cell adhesion assay

196 A total of $5 \times 10^{3}$ cells labeled with cell tracker were plated onto 96-well plates coated 197 with laminin, and the cells were cultured in RPMI-1640 medium with 5\% fetal bovine 198 serum at $37^{\circ} \mathrm{C}$ in an incubator with $5 \% \mathrm{CO}_{2}$ for 3 hours.

199

200 Chromatin immunoprecipitation (ChIP) assay

201 A total of $1 \times 10^{7} \mathrm{NIH} / 3$ T3-MARVELD1-V5 cells were used for the ChIP assay. ChIP was

202 performed as described previously (Weinmann and Farnham, 2002). Promoter 203 regions were PCR amplified with specific pairs of primers that are listed in Table s1.

204

205 Luciferase assay

206 HTR-8/SVneo cells were cultured in 12-well plates (Corning Glass, Tewksbury, MA, 207 USA) and were transfected with $1 \mu \mathrm{g}$ of PC or MARVELD1-V5 along with an integrin $208 \beta 4$ promotor construct. At 24 hours after transfection, luciferase activity was assayed 209 with $10 \mu \mathrm{l}$ of lysate using the Dual-Luciferase reporter assay system from Promega 210 (Madison, WI, USA) and a Dynex (Sunnyvale, CA, USA) luminometer. The transfection 211 efficiency was normalized to Renilla luciferase activity. Luciferase activity was 
212 measured using the CytoFluorplate 4000 Luminescence Microplate Reader (ABI,

213 Foster City, CA). Three transfection assays were performed to obtain statistically

214 significant data.

215

216 Statistical analysis

217 Statistical analyses were performed via two-tailed Student's t-test or one-way

218 ANOVA where indicated. $P<0.05$ was considered as statistically significant, $P^{*}<0.05$, $219 P^{* *}<0.01$, and $P^{* * *}<0.001$. Data from at least three independent experiments per 220 group were used for evaluation. Values are presented as the mean \pm SEM.

221 
Results

223

224 Generation of MARVELD1 knockout mice via the conditional Cre-loxP targeting 225 system

226 In humans, the nuclear factor MARVELD1 is a potential tumor suppressor. Our

227 previous study found that MARVELD1 is a novel protein and is downregulated in 228 multiple cancers via methylation of its promoter. More importantly, it mediates cell 229 adhesion and migration by regulating integrin family molecules. To further 230 investigate the role of this novel protein in developmental processes, we generated 231 the MARVELD1 knockout mice using a conditional targeting vector (Fig. 1A). We

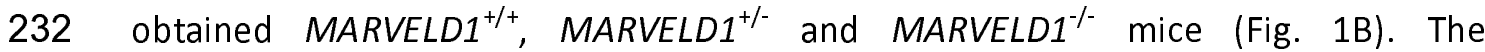
233 MARVELD1 $1^{-/}$mice are able to develop into normal adults.

234

MARVELD1 knockout mice have lower newborn offspring rate

236 The knockout of MARVELD1 does not result in a distinct phenotype after birth. We 237 used MARVELD1 $1^{-/}$mice to identify the phenotype of MARVELD1 deletion. At first, we 238 assessed MARVELD1 function by determining the birth rate of MARVELD1 $1^{+/-}$ 239 intercrossed mice. The newborn offspring genotypes of 68 litters were counted. 240 Notably, $0.3749 \pm 0.03082$ were wild-type, $0.4746 \pm 0.03068$ were heterozygotes, 241 and $0.1505 \pm 0.01998$ were homozygotes. Compared with the wild-type offspring, 242 the birth rate of homozygotes offspring was significantly lower $(P<0.001)$ (Fig. $1 C$ ). 243 We also collected MARVELD1 $1^{+/+}$intercross, MARVELD $1^{+/-}$intercross, and MARVELD $1^{-/-}$

244 intercross newborn offspring genotypes to confirm the results. Compared with the 245 wild-type intercross group, the birth rate in the homozygous group was significantly 246 lower $(P<0.01)$ (Fig. 1D); this data indicated that the MARVELD1 ${ }^{-1-}$ mice have lower 247 birth rates than that of wild-type mice. To further identify the reasons that impact 248 the knockout mice birth rate, we analyzed the E18.5 embryo numbers and the 249 newborn offspring pup numbers after intercrossing MARVELD1 ${ }^{-1-}$ mice; compared 250 with the E18.5 embryo numbers, the newborn offspring pup numbers were 
251 significantly lower ( $P<0.001)$ (Fig. 1E), which indicated an increased tendency to lose

252 pups before childbirth.

253

254 The pregnant MARVELD1 knockout mice exhibited a placenta accreta phenotype

255 In addition to decreased pup numbers in the MARVELD1 knockout mice after E18.5, 256 we also found that the placenta attached to the maternal uterus (Fig. 1F) and that

257 the newborn offspring exhibited long umbilical cords (Fig. 1G) by the time of birth.

258 These phenotypes suggested the MARVELD1 knockout mice, to a large extent, 259 exhibited placenta accreta. We further tested the expression levels of MARVELD1 in 260 the E18.5 wild-type mouse organs and found that MARVELD1 was highly expressed in 261 the placenta (Fig. 2A); additionally, the expression levels were increased in the 262 placentas from E10.5 to E18.5 (Fig. 2B), which is the time point that correlates with 263 the trophoblast cell invasion process. We further identified the localization of 264 MARVELD1 in wild-type E18.5 placentas. As shown in Fig. 2C, MARVELD1 was highly 265 expressed in the trophoblast cells, especially the trophoblast cells that tended to 266 migrate into the maternal decidua. These observations indicate that MARVELD1 plays 267 a role in trophoblast cell invasion.

268

269 The trophoblast cells of the MARVELD1 knockout mouse placenta exhibited 270 increased cell invasion

271 The high expression of MARVELD1 in the placenta and the trophoblast cells partially 272 revealed that MARVELD1 plays a role in trophoblast cell invasion. To further confirm 273 this hypothesis, we investigated trophoblast cell invasion in placenta accreta mice. 274 HE staining showed abnormal adhesion of the placenta to the myometrium (Fig. 3A). 275 Using immunohistochemical staining of Cytokeratin18, a trophoblast cell marker, we 276 observed that the maternal decidua was highly occupied by trophoblast cells in 277 placenta accreta mice (Fig. 3B). After we confirmed the placenta accreta phenotype, 278 the rate of placenta accreta was determined in MARVELD1-1- female mice. In total, 
$27945.95 \%$ of female MARVELD $1^{-/-}$mice exhibited the placenta accreta phenotype, and $28070.59 \%$ of those mice were pregnant for the first time (Fig. 3C). To double confirm 281 the over-invasion of trophoblast cells in MARVELD1 knockout placenta, the E18.5 282 placentas of wild-type mice and MARVELD1 knockout mice were compared. We used 283 HE staining to examine the architecture of the junction layer, which contain the 284 trophoblast cells that invade the maternal decidua. The boundary of the junction 285 layer in wild-type mice was clear; in contrast, the boundary in MARVELD1 knockout 286 mice was indistinct (Fig. 3D). Using immunohistochemical staining, we visualized a 287 clear difference of trophoblast cell invasion between E18.5 wild-type placenta and 288 MARVELD1 knockout placenta. An increase in trophoblast cell invasion from the 289 E18.5 knockout placentas into the maternal decidua was observed (Fig. 3E). These 290 observations confirmed a strong connection between MARVELD1 and the 291 over-invasion of trophoblast cells.

292

\section{MARVELD1 was downregulated during trophoblast cell migration and invasion}

294 To further elucidate the contribution of MARVELD1 during trophoblast cell invasion, 295 the HTR8/SVneo cell line was developed as an in vitro model. In human cell lines, the MARVELD1 expression level is higher in mesenchymal cell lines and is lower in

297 epithelial cell lines. The MARVELD1 expression level in the HTR8/SVneo cells was in 298 the mid-range (Fig. 4A). Ten ng/ $\mu \mathrm{L}$ TGF- $\beta 1$ was added to HTR8/SVneo cells, and 299 afterwards, the invasion ability of the HTR-8/SVneo cells was improved (Fig. 4B). 300 Then, we tested the expression of epithelial markers, mesenchymal markers, integrin $301 \beta 1$, integrin $\beta 4$, and MARVELD1. As shown in Fig. $4 \mathrm{C}$, treatment with $10 \mathrm{ng} / \mu \mathrm{L}$ TGF- $\beta 1$ 302 led to a significant decrease in E-cadherin expression level $(P<0.001)$ and an increase 303 in the expression of mesenchymal markers $\mathrm{N}$-cadherin and snail $(P<0.001)$. When the 304 invasion ability of HTR8/SVneo cells was improved, we detected a notable increase of 305 integrin $\beta 1$ and a significant decrease of integrin $\beta 4$; MARVELD1 was slightly but not 306 that significantly decreased. These results indicated that the HTR8/SVneo cell line 
was an ideal model to assess MARVELD1-mediated trophoblast cell invasion.

We further used the Transwell chamber to select cells by their migration ability. Cells above the membrane and below the membrane were collected separately. Different cell numbers were used: $2 \times 10^{4}, 4 \times 10^{4}, 6 \times 10^{4}$ and $8 \times 10^{4}$. MARVELD1 expression in the cells above the membrane and below the membrane was significantly different at each cell density except $8 \times 10^{4}$ (Fig. 4D). Different time points were also tested by plating $6 \times 10^{4}$ cells onto the chamber and collecting cells above the membrane and below the membrane after $0,18,22,26$, and 36 hours. The difference in MARVELD1 expression between the migrated cells and the non-migrated cells exhibited the highest difference at 18 hours, and the difference in expression diminished afterwards; there was no significant difference after 36 hours. These data indicate that MARVELD1 is expressed at low levels in the migrated trophoblast cells.

\section{MARVELD1 regulated trophoblast cell invasion via integrin $\beta 4$}

In human non-small cell lung cancer, we found that MARVELD1 mediated the balance of integrin $\beta 1 / \beta 4$ and the EMT phenotype(Yao et al., 2015). Since integrin $\beta 1 / \beta 4$ belongs to integrin family, which participates in trophoblast cell invasion, we transfected the HTR8/SVneo cells with siRNAs that can downregulate the mRNA expression level of MARVELD1 to assess if MARVELD1 regulates the expression of integrin $\beta 1 / \beta 4$ in trophoblast cells. We speculated that MARVELD1 had the same roles in trophoblast cells as in human non-small cell lung cancer. We found, however, that the roles were not the same, but they were similar. Compared with the scrambled siRNA-transfected HTR8/SVneo cells, MARVELD1 siRNA significantly decreased the mRNA expression level of MARVELD1 $(P<0.001)$ as well as the expression level of integrin $\beta 4(P<0.001)$, but the expression level of integrin $\beta 1$ was not significantly changed (Fig. 5A). The protein level of integrin $\beta 1 / \beta 4$ correlated with the mRNA results (Fig. 5B, 5C). These results indicated that MARVELD1 specifically 
335 regulated the expression of integrin $\beta 4$ in trophoblast cells. To determine whether 336 this relationship between MARVELD1 and integrin $\beta 4$ was involved in the migration 337 and invasive ability of HTR8/SVneo cells, the cells were transfected with scrambled 338 siRNA, siRNA for MARVELD1, vector, siRNA for MARVELD1 and vector, integrin $\beta 4$, 339 and siRNAs for MARVELD1 and integrin $\beta 4$. The migration and invasive abilities were 340 positively correlated, and the abilities from highest to lowest were as follows: 341 siMARVELD1 siMARVELD1 +PC; scrambled siRNA scrambled siRNA +PC; integrin $\beta 4$ $342+$ siMARVELD1; and integrin $\beta 4$. Compared with the scrambled siRNA group, the 343 migration and invasive abilities were enhanced in the cells transfected with siRNA for 344 MARVELD1 (Fig. 5D). These results support the synergy between MARVELD1 and 345 integrin $\beta 4$ in the invasion of HTR8/SVneo cells.

$346 \quad$ Since integrin $\beta 4$ promoted its signaling events and mediated cell adhesion to 347 ECM, we also tested the adhesion ability of cells transfected with scrambled siRNA, 348 SIRNA for MARVELD1, vector, siRNA for MARVELD1 and vector, integrin $\beta 4$, and 349 siRNAs for MARVELD1 and integrin $\beta 4$. The adhesion ability of each group was 350 negatively correlated with their migration and invasive abilities (Fig. 5E). These 351 results further support that MARVELD1 regulates trophoblast cell invasion via 352 integrin $\beta 4$-dependent cell adhesion.

353 As the invasion process of trophoblast cell is somewhat like an EMT process, 354 EMT markers were also tested to double confirm our hypothesis. As shown is Fig. 5F, 355 the E marker was down regulated when the invasive ability was increased, and there 356 were no significant changes in the $M$ marker. These data fully confirm our previous 357 hypothesis.

MARVELD1 increases the expression of integrin $\beta 4$ by enhancing its promoter 360 activity

361 As the expression of integrin $\beta 4$ is correlated with MARVELD1, this raises the 362 question of how MARVELD1 regulates the expression of integrin $\beta 4$. We have 
363 demonstrated before that MARVELD1 could specifically bind to the promoter of 364 integrin $\beta 4$ and activate it, which then enhanced the expression of integrin $\beta 4$ in 365 human cancer cell lines. To determine whether the same mechanism occurs in 366 mouse cells, NIH/3T3-MARVELD1-V5 cells were used in a ChIP assay. As shown in Fig. 3676 A, MARVELD1 specifically bound to the integrin $\beta 4$ promoter. Luciferase assays were performed in HTR8/SVneo cells transfected with MARVELD1-V5 plasmids. As shown

369 in Fig. 6B, the cells transfected with MARVELD1-V5 resulted in significantly higher 370 integrin $\beta 4$ promoter activity than PC control cells. These data demonstrate that 371 MARVELD1 regulates the expression of integrin $\beta 4$ by enhancing its promoter 372 activity.

373

Loss of MARVELD1 affects trophoblast cell invasion via integrin $\beta 4$ expression

376 The migration capability of HTR8/SVneo cells could be affected by the decreased 377 expression of MARVELD1 via its effects on the integrin $\beta 4$-mediated cell adhesion 378 pathway, thus explaining the over-invasiveness of trophoblast cells observed in 379 MARVELD1 knockout mice in vivo. The invasion of trophoblast cells is a precise 380 process that is regulated by complex molecules; therefore, we aimed to further 381 confirm the molecular mechanisms in vivo. In E18.5 placentas, the deletion of 382 MARVELD1 resulted in a decrease of the integrin $\beta 4$ (Fig. 6C) expression, 383 demonstrating an overall increase in its cell adhesion pathway. In Fig. 6D, we show in 384 E18.5 MARVELD1 ${ }^{-/}$placentas that there was a large amount of trophoblast cells that 385 invaded into the maternal decidua compared to wild-type mice. The observed effect 386 of MARVELD1 knockout in the E18.5 placenta supports the notion that the deletion 387 of MARVELD1 downregulates the expression of integrin $\beta 4$ and promotes the 388 over-invasion of trophoblast cells (Fig. 7A). 


\section{Discussion}

392

393 The function of MARVELD1, a novel nuclear factor that mediates cell adhesion and

394 migration in lung cancer, in mouse development is still unclear. Since gene knockout

395 mice are ideal models in which to study gene function, we developed the MARVELD1 knockout mouse to assess phenotypes after gene deletion, and more importantly, to

397 explore the molecular mechanisms of this novel protein. Our data indicated that the 398 knockout of MARVELD1 caused low birth rates but did not affect the numbers of embryonic offspring. We identified a placenta accreta phenotype, characterized by

400 the attachment of the placenta to the maternal uterus, and the over-invasion of 401 trophoblast cells in MARVELD1 knockout mice. Consistent with these observations, we found that the loss of MARVELD1 suppressed integrin $\beta 4$ expression and the 403 integrin $\beta 4$-dependent cell adhesion process, demonstrating that MARVELD1 404 mediates integrin $\beta 4$ expression and plays important roles in placenta development, 405 especially in trophoblast cell invasion (Fig. 7A, 7B).

406 MARVELD1 is a novel MARVEL domain-containing protein that is downregulated in 407 multiple human cancers(Wang et al., 2009). The MARVEL-domain containing proteins 408 such as MYADM(Aranda et al., 2011) and occludin(Huber et al., 2000) are involved in 409 cell adhesion and migration. Our previous studies have revealed that MARVELD1 410 regulates integrin $\beta 1$ though a pre-mRNA processing pathway(Wang et al., 2013) and 411 that MARVELD1 is involved in TGF- $\beta 1$-mediated EMT by regulating the balance of 412 integrin $\beta 1$ and $\beta 4$ expression in NSCLC cells(Yao et al., 2015). These findings indicate 413 that MARVELD1 plays a role in cell adhesion and migration in cancers. Since adhesion 414 and migration are basic cell characteristics, MARVELD1 is highly conserved between 415 different species, and therefore, we hypothesized that MARVELD1 plays a role during 416 mouse development.

417 To test the hypothesis, MARVELD1 knockout mice were developed. The 418 MARVELD1 $^{-/}$mice can develop into normal adults but have lower birth rates 
419 compared to wild-type mice. During the birthing process in MARVELD1 knockout

420 mice, we visualized that the placentas were difficult to detach from the maternal

421 uterus due to the over-invasion of trophoblast cells. Cell migration is central to

422 multiple physiological processes, including embryonic development and cancer

423 metastasis. During both development and normal homeostasis, cells interact with

424 each other and the surrounding environment, including morphogen and the ECM, to

425 maintain tissue structure, organization and function(Paluch et al., 2016). During

426 embryonic development, cells migrate through interacting with their surrounding

427 environment; the tissue remodeling and morphogenesis processes involve the

428 migration of different cell types (Muthuswamy and Xue, 2012). The migration of cells

429 engaged in tissue-remodeling events contribute to cell invasion during development.

430 Rodents and human have similar hemochorial placenta, and trophoblast cell invasion

431 plays important roles in this type of placenta. The trophoblast cell invades into the

432 maternal uterus in search of spiral arterioles and veins. Trophoblast cells breach the

433 spiral arterioles and replace the endothelial and smooth muscle cells (Red-Horse et

434 al., 2006). These processes are important for both proper fetal perfusion and

435 attachment(Maltepe and Fisher, 2015). Previous studies have indicated that the

436 trophoblast cell invasion process is similar to epithelial-to-endothelial

437 transition(Maltepe and Fisher, 2015); E-cadherin and integrin $\alpha 6 \beta 4$ are

438 downregulated during trophoblast cell invasion(Maltepe and Fisher, 2015).

439 Here, we identified that the downregulation of MARVELD1 decreases the 440 expression of integrin $\beta 4$ and integrin $\beta 4$-dependent cell adhesion process both in 441 vivo and vitro, which correlates with the phenotype observed in MARVELD1 442 knockout mice. These in vivo and in vitro studies further elucidated the contribution 443 of MARVELD1 in placenta development. Notably, previously reported findings mainly 444 highlighted integrin $\beta 4$ as an epithelial marker, and its expression was an indicator for 445 EMT. Based on our results, we suggest that integrin $\beta 4$ is also an EMT initiator, and 446 this process is also under the control of MARVELD1 during placenta development. 
447 Our study suggests a sequence of events that result in the over-invasion of 448 trophoblast cells; additional studies are required to further illustrate this process. 449 Although a lot has been discovered about the placenta in recent decades, there is a 450 lot more regarding placental abnormalities and pregnancy complications that remain 451 to be discovered. In summary, our study has elucidated a novel function of the 452 MARVELD1 gene in trophoblast cell invasion.

453

454 


\section{Acknowledgements}

456 The HTR8/SVneo cell line was a gift from Prof. Yongjun Yang (Jilin University). This 457 work was supported by the National Natural Science Foundation of China No. 45831571323 and Shenzhen Municipal Basic Science Foundation No. 459 JCYJ20140417173156097 to YL; the China Postdoctoral Science Foundation No. $4602015 M 571426$ to YC.

461

462 Author contributions

$463 \mathrm{YL}$ and $\mathrm{YC}$ conceived the idea, designed experiments, provided the conceptual 464 framework for the study, and wrote the manuscript. $\mathrm{HZ}$ further contributed to 465 manuscript preparation. $\mathrm{YC}, \mathrm{HZ}, \mathrm{FH}, \mathrm{LY}$, and $\mathrm{CQ}$ performed the experiments. $\mathrm{YC}, \mathrm{HZ}$, $466 \mathrm{YZ}$ and $\mathrm{WL}$ analyzed the data, PD contributed to conceptual evaluation of the project. 467 
468

469

470

471

472

473

474

475

476

477

478

479

480

481

482

483

484

485

486

487

488

489
References

Anacker, J., Segerer, S. E., Hagemann, C., Feix, S., Kapp, M., Bausch, R. and Kammerer, U. (2011). Human decidua and invasive trophoblasts are rich sources of nearly all human matrix metalloproteinases. Mol Hum Reprod 17, 637-652.

Aranda, J. F., Reglero-Real, N., Kremer, L., Marcos-Ramiro, B., Ruiz-Saenz, A., Calvo, M., Enrich, C., Correas, I., Millan, J. and Alonso, M. A. (2011). MYADM regulates Rac1 targeting to ordered membranes required for cell spreading and migration. Mol Biol Cell22, 1252-1262.

Ball, E., Bulmer, J. N., Ayis, S., Lyall, F. and Robson, S. C. (2006). Late sporadic miscarriage is associated with abnormalities in spiral artery transformation and trophoblast invasion. The Joumal of pathology 208, $535-542$.

Bischof, P., Meisser, A. and Campana, A. (2000). Paracrine and autocrine regulators of trophoblast invasion--a review. Placenta 21 Suppl A, S55-60.

Damsky, C. H., Fitzgerald, M. L. and Fisher, S. J. (1992). Distribution patterns of extracellular matrix components and adhesion receptors are intricately modulated during first trimester cytotrophoblast differentiation along the invasive pathway, in vivo. J Clin Invest 89, 210-222.

Damsky, C. H., Librach, C., Lim, K. H., Fitzgerald, M. L., McMaster, M. T., 
490

491

492

493

494

495

496

497

498

499

500

501

502

503

504

505

506

507

508

509
Janatpour, M., Zhou, Y., Logan, S. K. and Fisher, S. J. (1994). Integrin switching regulates normal trophoblast invasion. Development 120 , $3657-3666$

Dashraath, P. and Lin, H. Z. (2016). Placenta Increta. N Engl J Med 375, 1382.

Fitzgerald, J. S., Poehlmann, T. G., Schleussner, E. and Markert, U. R. (2008). Trophoblast invasion: the role of intracellular cytokine signalling via signal transducer and activator of transcription 3 (STAT3). Hum Reprod Update 14, 335-344.

Godbole, G., Suman, P., Gupta, S. K. and Modi, D. (2011). Decidualized endometrial stromal cell derived factors promote trophoblast invasion. Fertil Steril 95, 1278-1283.

Gude, N. M., Roberts, C. T., Kalionis, B. and King, R. G. (2004). Growth and function of the normal human placenta. Thromb Res 114, 397-407.

Huber, D., Balda, M. S. and Matter, K. (2000). Occludin modulates transepithelial migration of neutrophils. J Biol Chem 275, 5773-5778.

Huppertz, B. (2008). Placental origins of preeclampsia: challenging the current hypothesis. Hypertension 51, 970-975.

Hustin, J., Jauniaux, E. and Schaaps, J. P. (1990). Histological study of the materno-embryonic interface in spontaneous abortion. Placenta 11, 477-486.

Irving, J. A., Lysiak, J. J., Graham, C. H., Hearn, S., Han, V. K. and Lala, P. K. 
(1995). Characteristics of trophoblast cells migrating from first trimester chorionic villus explants and propagated in culture. Placenta 16, 413-433.

514 Jauniaux, E., Collins, S. L., Jurkovic, D. and Burton, G. J. (2016). Accreta placentation: a systematic review of prenatal ultrasound imaging and grading of villous invasiveness. Am J Obstet Gynecol215, 712-721.

517 Khong, T. Y., De Wolf, F., Robertson, W. B. and Brosens, I. (1986). Inadequate maternal vascular response to placentation in pregnancies complicated by pre-eclampsia and by small-for-gestational age infants. Br J Obstet Gynaecol 93, 1049-1059.

521 Khong, T. Y. and Robertson, W. B. (1987). Placenta creta and placenta praevia creta. Placenta 8, 399-409.

523 Kim, Y. M., Bujold, E., Chaiworapongsa, T., Gomez, R., Yoon, B. H., Thaler, H.

524 T., Rotmensch, S. and Romero, R. (2003). Failure of physiologic transformation of the spiral arteries in patients with preterm labor and intact membranes. Am J Obstet Gynecol189, 1063-1069.

527 Lash, G. E. (2015). Molecular Cross-Talk at the Feto-Maternal Interface. Cold 528 Spring Harbor perspectives in medicine 5.

529 Luke, R. K., Sharpe, J. W. and Greene, R. R. (1966). Placenta accreta: the adherent or invasive placenta. Am J Obstet Gyneco/95, 660-668.

531 Maltepe, E. and Fisher, S. J. (2015). Placenta: the forgotten organ. Annu Rev 
533 Muthuswamy, S. K. and Xue, B. (2012). Cell polarity as a regulator of cancer cell behavior plasticity. Annu Rev Cell Dev Biol28, 599-625.

535 Paluch, E. K., Aspalter, I. M. and Sixt, M. (2016). Focal Adhesion-Independent Cell Migration. Annu Rev Cell Dev Bio/ 32, 469-490.

Pijnenborg, R., Anthony, J., Davey, D. A., Rees, A., Tiltman, A., Vercruysse, L. and van Assche, A. (1991). Placental bed spiral arteries in the hypertensive disorders of pregnancy. $\mathrm{Br} J$ Obstet Gynaecol 98, 648-655.

541 Red-Horse, K., Rivera, J., Schanz, A., Zhou, Y., Winn, V., Kapidzic, M., 542 Maltepe, E., Okazaki, K., Kochman, R., Vo, K. C., et al. (2006). 543 Cytotrophoblast induction of arterial apoptosis and lymphangiogenesis 544 in an in vivo model of human placentation. $J$ Clin Invest 116, 2643-2652.

546 Rossant, J. and Cross, J. C. (2001). Placental development: lessons from mouse mutants. Nature reviews. Genetics 2, 538-548.

548 Ruoslahti, E. (1991). Integrins. J Clin Invest 87, 1-5.

549 -- (1996). RGD and other recognition sequences for integrins. Annu Rev Cell Dev Biol12, 697-715.

551 Wang, S., Hu, J., Yao, Y., Shi, M., Yue, L., Han, F., Zhang, H., He, J., Liu, S. and $\mathrm{Li}, \mathrm{Y}$. (2013). MARVELD1 regulates integrin beta1-mediated cell 
adhesion and actin organization via inhibiting its pre-mRNA processing. Int J Biochem Cell Biol 45, 2679-2687.

Wang, S., Li, Y., Han, F., Hu, J., Yue, L., Yu, Y., Zhang, Y., He, J., Zheng, H., Shi, S., et al. (2009). Identification and characterization of MARVELD1, a novel nuclear protein that is down-regulated in multiple cancers and silenced by DNA methylation. Cancer Lett 282, 77-86.

559 Watson, E. D. and Cross, J. C. (2005). Development of structures and transport functions in the mouse placenta. Physiology (Bethesda) 20, 180-193.

562 Weinmann, A. S. and Farnham, P. J. (2002). Identification of unknown target genes of human transcription factors using chromatin immunoprecipitation. Methods 26, 37-47. MARVELD1 modulates cell surface morphology and suppresses epithelial-mesenchymal transition in non-small cell lung cancer. Molecular carcinogenesis. Identification of mouse MARVELD1 as a microtubule associated protein that inhibits cell cycle progression and migration. Molecules and cells 
bioRxiv preprint doi: https://doi.org/10.1101/106807; this version posted February 7, 2017. The copyright holder for this preprint (which was not certified by peer review) is the author/funder. All rights reserved. No reuse allowed without permission.

575 
576

577

578

579

580

581

582

583

584

585

586

587

588

589

590

591

592

593

594

595

596

597

598

599

600

601

602

603

604

\section{Figure legends}

Figure 1. MARVELD1 knockout mice exhibit birthing difficulties.

(A) Strategy for the modification of the MARVELD1 locus in ES cells and mice. Shown in this figure is a schematic diagram of the targeting vector, the wild-type locus, and the modified MARVELD1 locus before and after excision of the PGK-Neo cassette with Cre recombinase.

(B) Genotype analysis of DNA from the tails of offspring animals for the modified MARVELD1 allele. PCR reactions were carried out with primer pairs specific for the wild-type MARVELD1 locus and the MARVELD1 knockout locus from which the neo cassette had been excised by breeding with Cre mice (see text and Materials and Methods for details). The sizes of the expected PCR products are indicated on the right side, and the genotypes are indicated above each lane.

(C) Genotype distribution of progeny in MARVELD $1^{+-}$intercross litters, $\mathrm{n}=68$.

(D) Numbers of progeny in MARVELD1 $1^{+/+}(\mathrm{n}=21)$, MARVELD $^{+/-}(\mathrm{n}=42)$, MARVELD1 $^{-/-}$ $(n=23)$ intercross litters.

(E) Numbers of progeny in MARVELD $1^{-/}$intercross litters before birth $(n=18)$ and after birth $(n=23)$.

(F) Difficult birth phenotype of the MARVELD1 $1^{-/}$mother, and the successful production of the wild-type mother.

(G) The long umbilical cord phenotype of newborn offspring from the MARVELD1 $1^{-1}$ intercross.

Figure 2. MARVELD1 is highly expressed in the placenta and invasive trophoblast cells.

(A) Relative expression of the mRNA level encoding MARVELD1 in E18.5 organs, including brain, heart, liver, lung, kidney and placenta, was determined by real-time RT-PCR. GAPDH mRNA was used to normalize the variability in template loading. The data are reported as the mean \pm SEM. 
605 (B) Relative expression of mRNA encoding MARVELD1 in E10.5, E11.5, E12.5, E15.5 606 and E18.5 placentas.

607 (C) Immunohistochemical analysis of E18.5 placenta show that MARVELD1 is highly 608 expressed in the placenta and invasive trophoblast cells.

609

Figure 3. MARVELD1/- placenta exhibits abnormal architecture and trophoblast cell

\section{1 over-invasion}

612 (A) HE staining showing the attachment of the placenta to the maternal uterus from 613 a MARVELD1 KO dam.

614 (B) Immunohistochemistry of placentas from MARVELD1 KO dams for Cytokeratin18, 615 a trophoblast cell marker.

616 (C) The occurrence the dystocia phenotype in MARVELD $1^{-/-}$female mice, $\mathrm{n}=37$; the 617 relationships between the dystocia phenotype and the times of pregnancy in $618 M A R V E L D 1^{-/}$female mice, $\mathrm{n}=17$.

619 (D) HE staining of the placenta from E18.5 MARVELD1 KO and control dams showing 620 the abnormal architecture of the junctional region in MARVELD1 $1^{-1-}$ placenta.

621 (E) Immunohistochemistry of the placenta from E18.5 MARVELD1 $1^{-/}$and control dams 622 for Cytokeratin18. Note the over-invasion of trophoblast cells in the MARVELD1 1 623 placenta.

624

Figure 4. MARVELD1 and integrin $\beta 4$ are downregulated in HTR8/SVneo cells during

626 EMT

627 (A) Relative expression of MARVELD1 mRNA in human cell lines was determined by 628 real-time RT-PCR. GAPDH mRNA was used to normalize the variability in template 629 loading. The data are reported as the mean \pm SEM.

630 (B) For the Transwell assay, $10 \mathrm{ng} / \mu \mathrm{L}$ TGF- $\beta 1$ was added to the cells, and the images 631 were acquired after 24 hours.

632 (C) The mRNA levels of epithelial markers, mesenchymal markers, integrin $\beta 1$, 
633 integrin $\beta 4$, and MARVELD1 with/without TGF- $\beta 1$ determined by real-time RT-PCR.

634 (D) The mRNA level of MARVELD1 during cell migration stratified by cell number and 635 migration time. Non-migrated cells above the chamber and migrated cells below the 636 chamber were collected separately.

637

638 Figure 5. MARVELD1 regulates trophoblast cell invasion via the integrin $639 \quad$ B4-mediated cell adhesion pathway

640 SiRNA for MARVELD1 was added to cells to downregulate MARVELD1 mRNA 641 expression levels.

642 (A) Relative expression of integrin $\beta 1$, integrin $\beta 4$, and MARVELD1 mRNA in cells was 643 determined by real-time RT-PCR. GAPDH mRNA was used to normalize the variability 644 in template loading.

645 (B) Western blot analysis of the expression of integrin $\beta 1$ and integrin $\beta 4$. GAPDH 646 was used as a loading control.

647 (C) Images of cells immunostained with antibodies against integrin $\beta 4$.

648 (D) Cells were labeled with cell tracker, and cell adhesion ability was identified after 3 649 hours.

650 (E) Transwell assay to analyze cell migration and invasion.

651 (F) Relative expression of the mRNA level encoding MARVELD1, integrin $\beta 4$, 652 E-cadherin and N-cadherin in cells. As determined by real-time RT-PCR, GAPDH 653 mRNA was used to normalize the variability in template loading.

654

655 Figure 6. MARVELD1 increases the expression of integrin $\beta 4$ by enhancing its 656 promoter activity

657 (A) A ChIP assay was performed by using HTR8/SVneo cell lysate. Chromatin was 658 immunoprecipitated using a V5-tag specific antibody. PCR was carried out using 659 specific primers for the integrin $\beta 4$ promoter.

660 (B) Cells were transfected with the integrin $\beta 4(-1,594 \sim-449)$ or the integrin $\beta 4$ 
$661(-1,594 \sim-1,418)$ luciferase reporter plasmid. The firefly luciferase activity was

662 measured 24 hours post-transfection and normalized to Renilla luciferase activity.

663 Values represent the mean \pm SEM of three independent experiments.

664 (C) Embryonic side: images of E18.5 MARVELD1 ${ }^{+/+}$and MARVELD1/- placenta sections 665 immunostained with antibodies against integrin $\beta 4$ (green) and Cytokeratin18 (red) 666 as well as DAPI staining (blue).

667 (D) Maternal side: images of E18.5 MARVELD1 $1^{+/+}$and $M A R V E L D 1^{-/}$placenta sections 668 immunostained with antibodies against integrin $\beta 4$ (green) and Cytokeratin18 (red) 669 as well as DAPI staining (blue).

670

671 Figure 7. Proposed models for MARVELD1-mediated trophoblast cell invasion in 672 vitro and in vivo

673 (A) Working model of MARVELD1 in mediating in vivo trophoblast cell invasion.

674 When MARVELD1 is knocked out, the expression level of integrin $\beta 4$ is 675 downregulated and the adhesive ability of cells is suppressed, which boost cell 676 migration and invasion, leading to trophoblast cell over-invasion and the placenta 677 accreta phenotype in MARVELD1/- mice.

678 (B) Underlying molecular mechanisms. MARVELD1 binds to the integrin $\beta 4$ promoter 679 to activate its transcription. Then, integrin $\beta 4$ upregulates the signaling processes 680 that facilitate cell adhesion and suppress cell migration and invasion. 


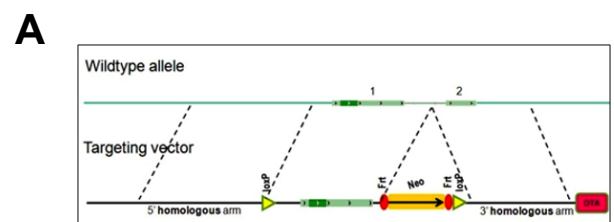

B Targeted allele

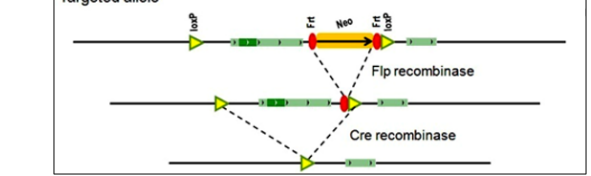

C

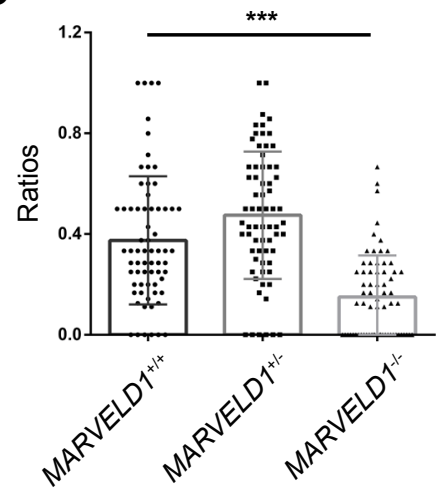

D

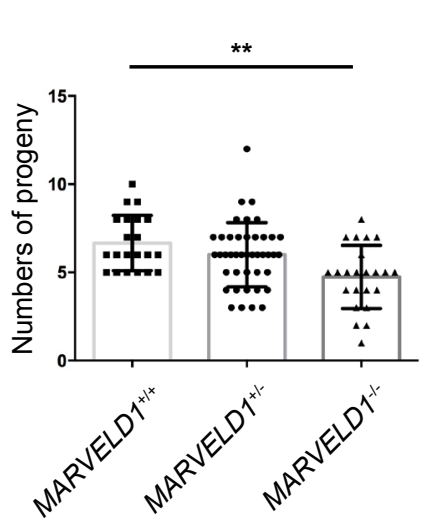

E

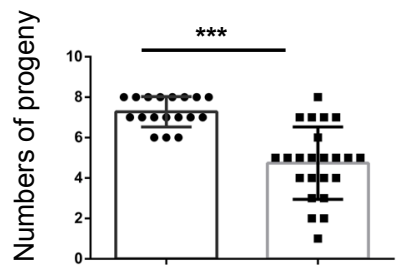

F

MARVELD1/++ MARVELD1/-

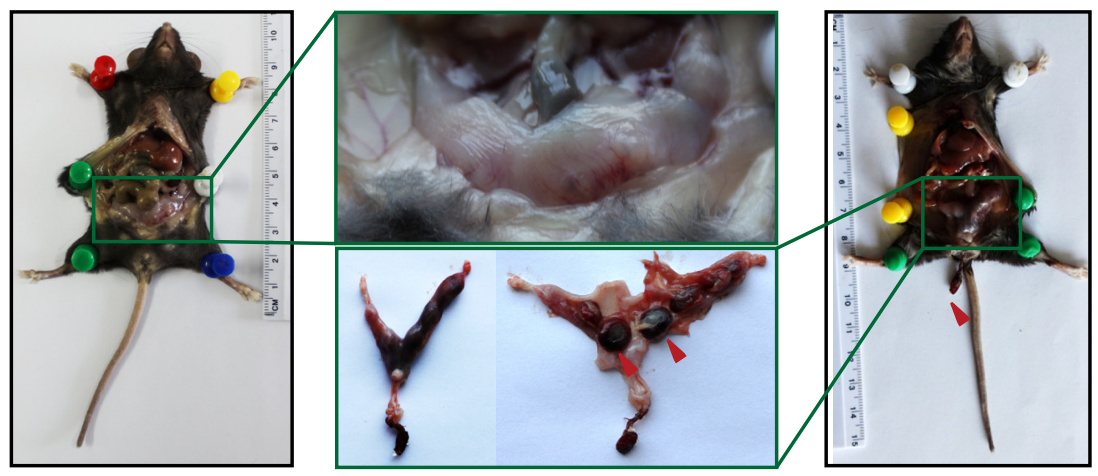

G MARVELD1/++

MARVELD1--
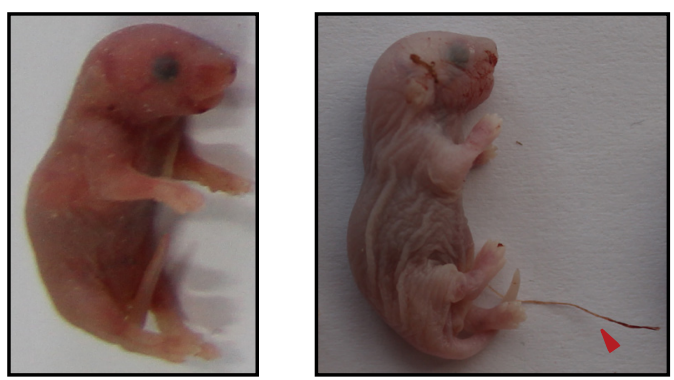
A

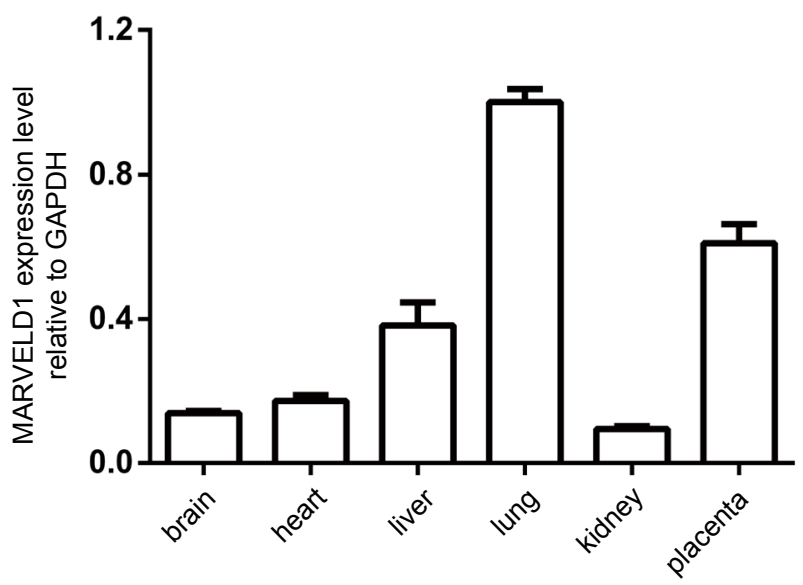

B

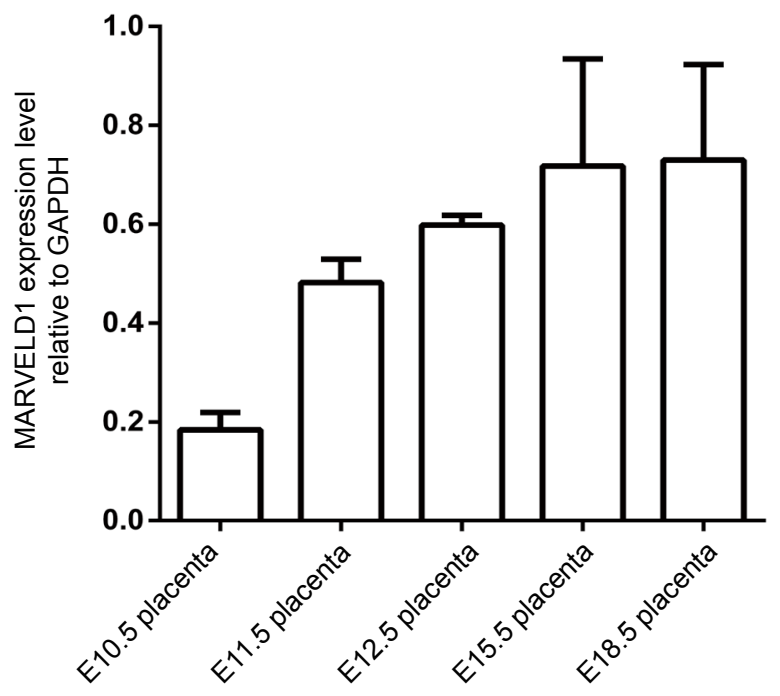

C

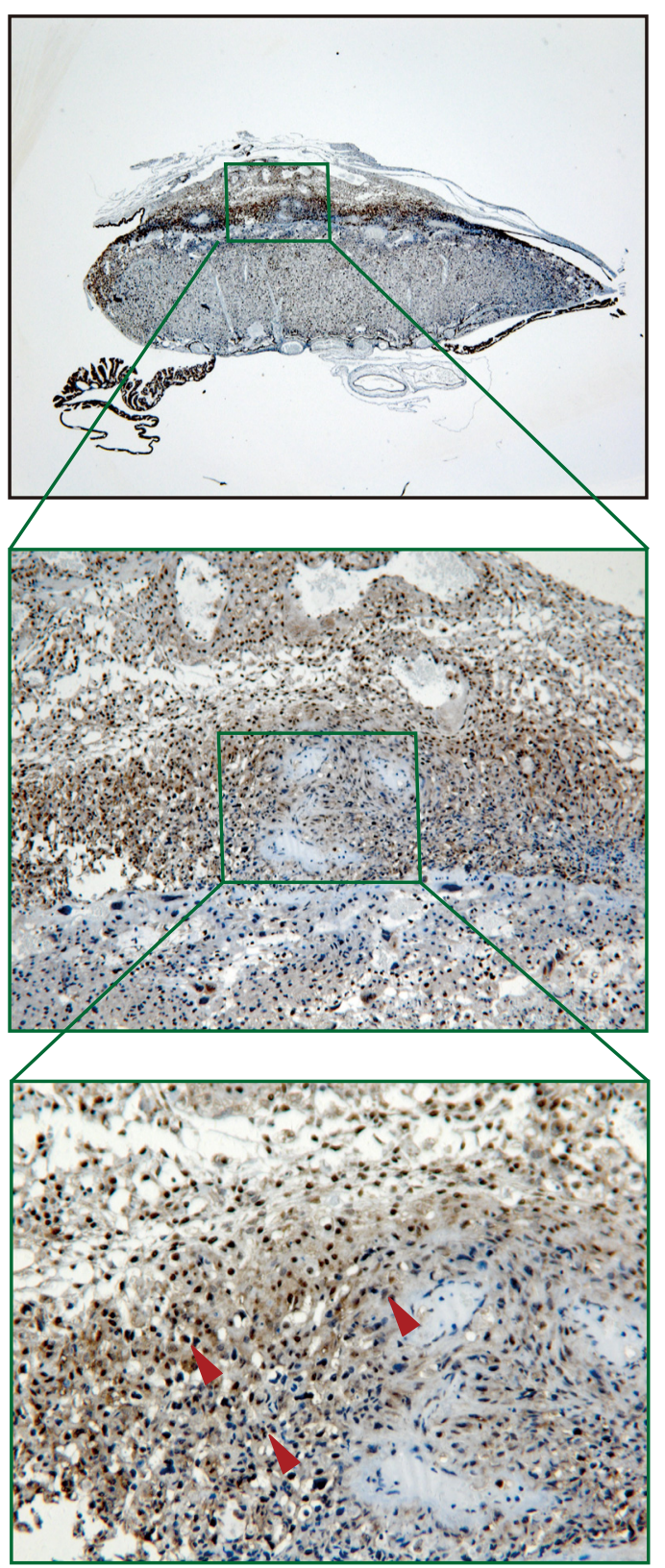


A
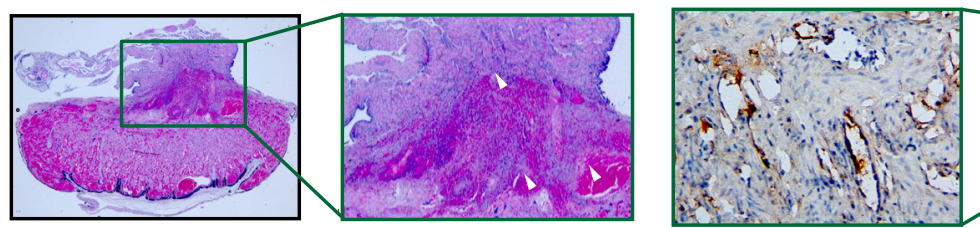
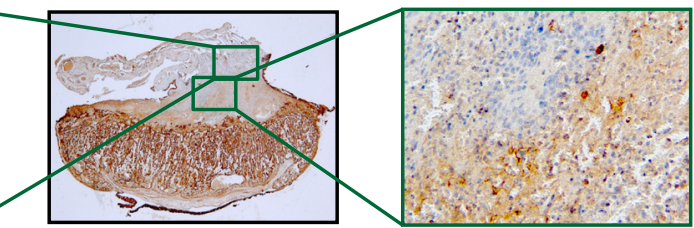

C

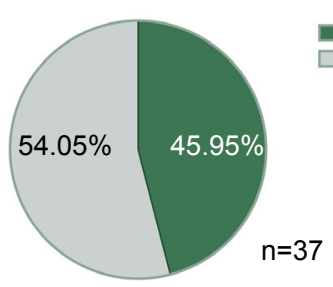

Placenta accreta rate

Successful pregnancy rate

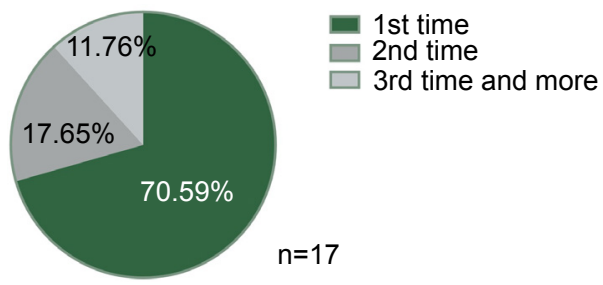

D

MARVELD $1^{1 /+}$

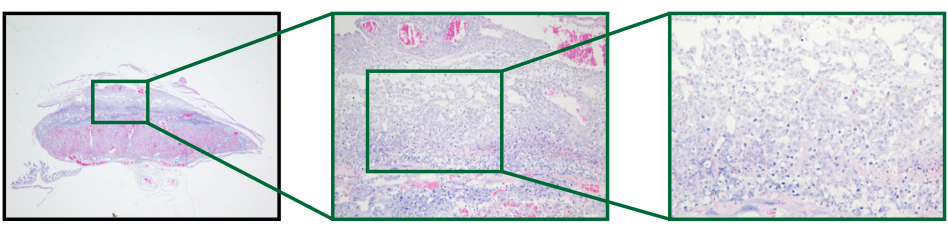

MARVELD $1^{-1-}$

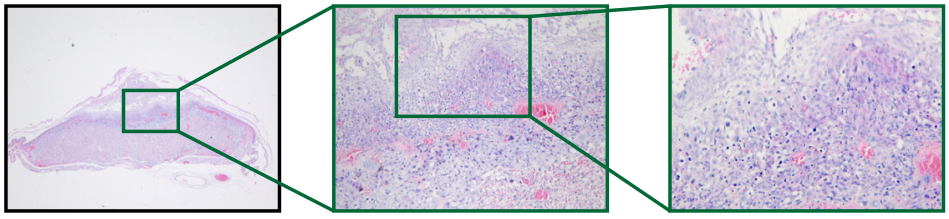

E

MARVELD $1^{1 /+}$

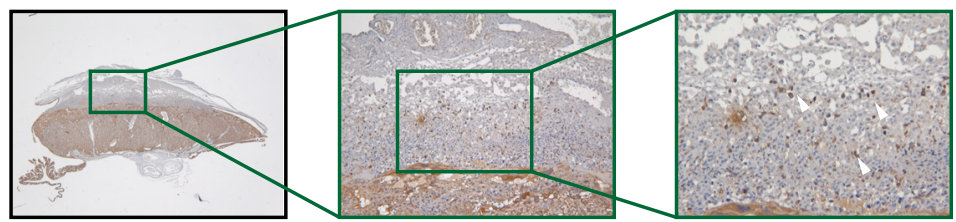

MARVELD1-

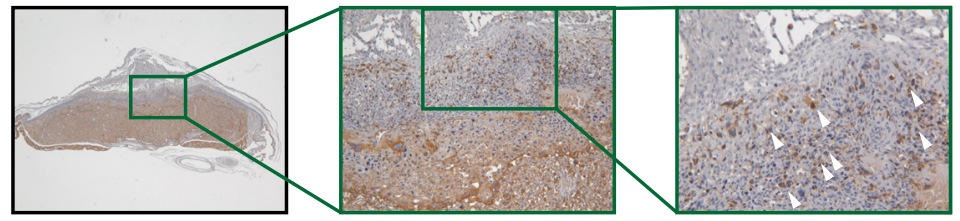


A

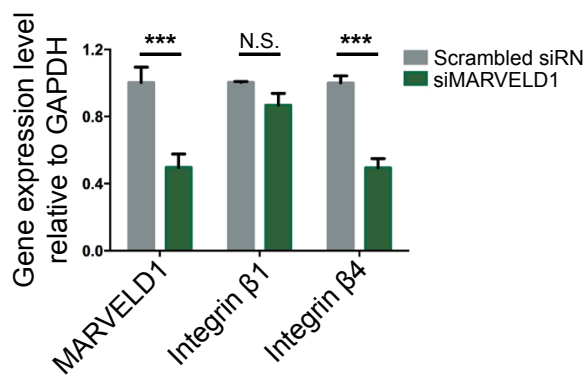

B

- - Integrin $\beta 4$ Scrambled siRNA

$\Longrightarrow-$ Integrin $\beta 1$

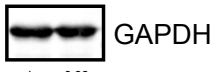

C

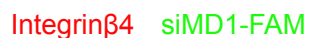

DAPI Merge

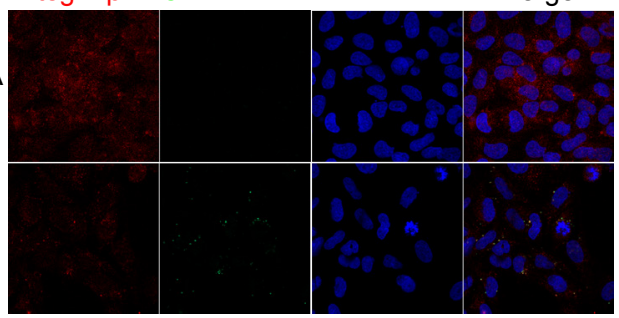

D

Scrambled siRNA siMARVELD1

PC

SIMARVELD1+PC

Integrin $\beta 4$ Integrin $\beta 4+$ siMARVELD1

Invasion

Migration

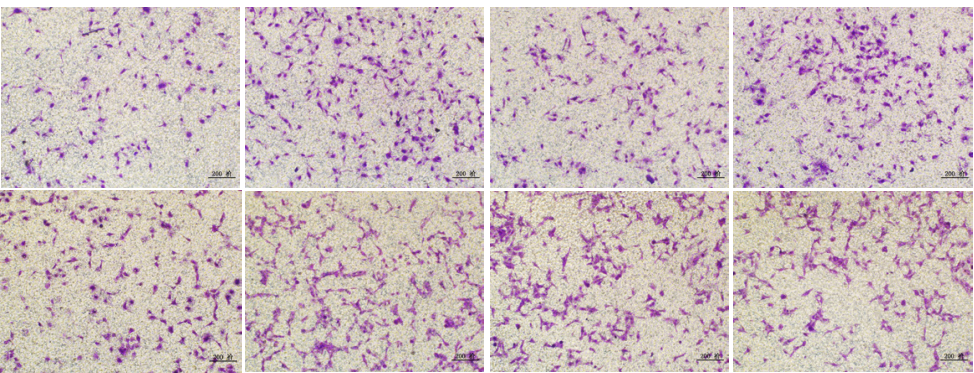

E
Scrambled siRNA siMARVELD1

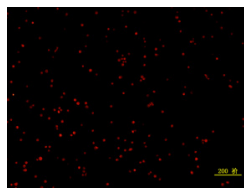

Before wash

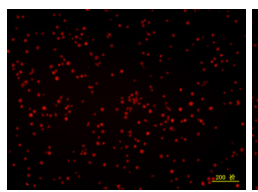

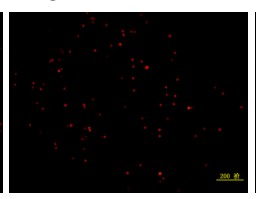

PC

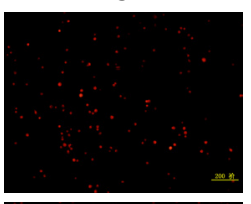

SiMARVELD1+PC

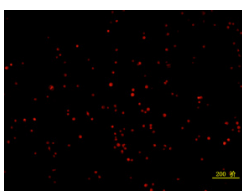

Integrin $\beta 4$ Integrin $\beta 4+$ siMARVELD1
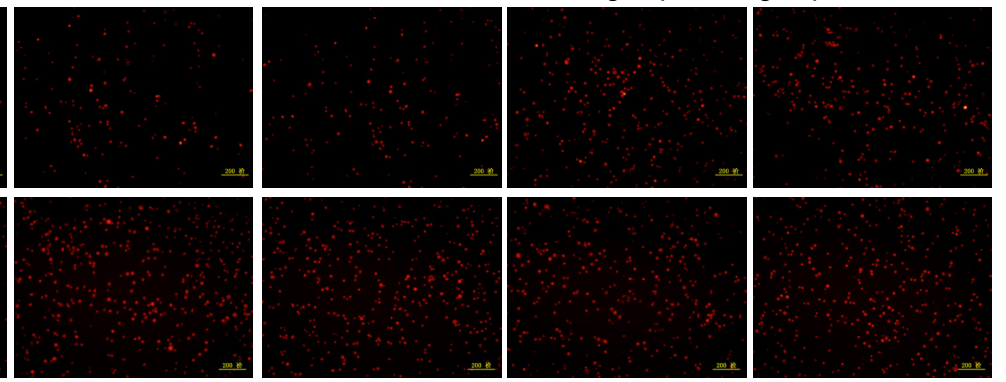

F
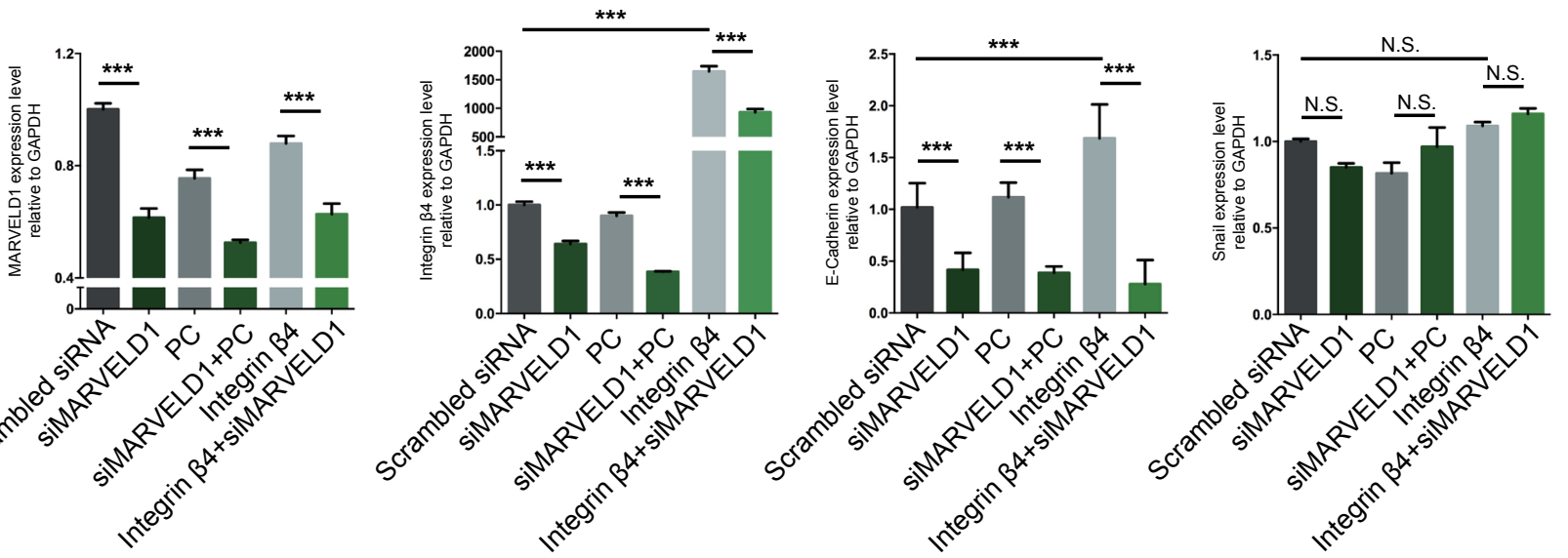

Figure 5 


\section{A}

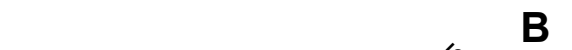

C

\section{Embyonic side}

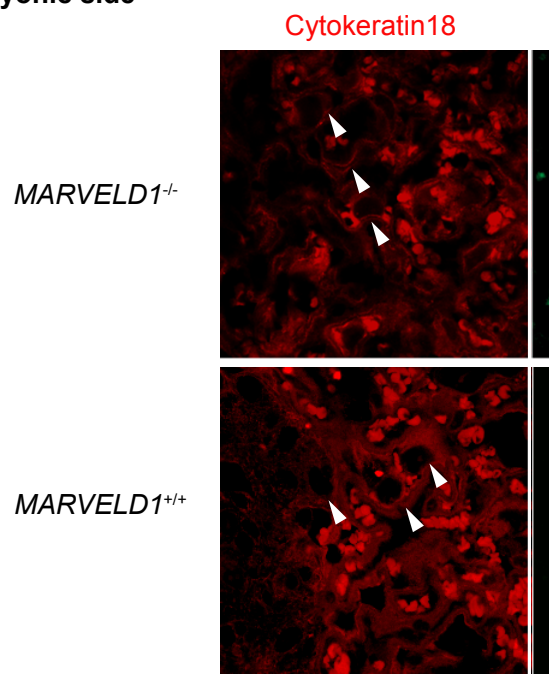

D

\section{Maternal side}

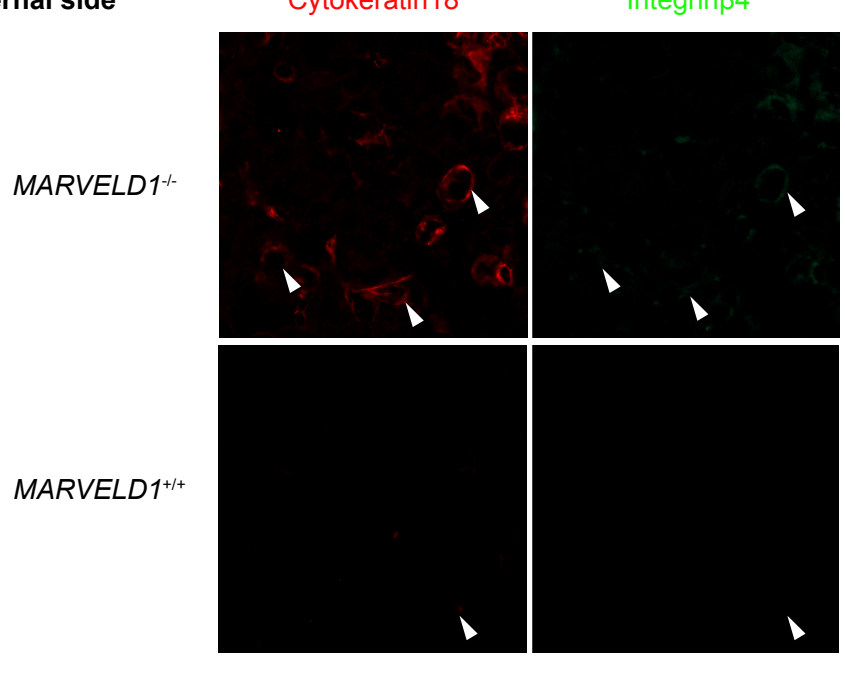

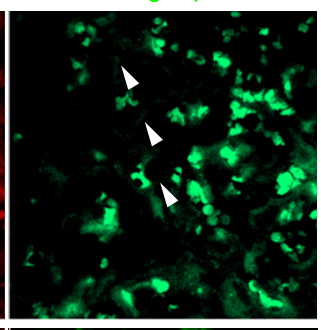
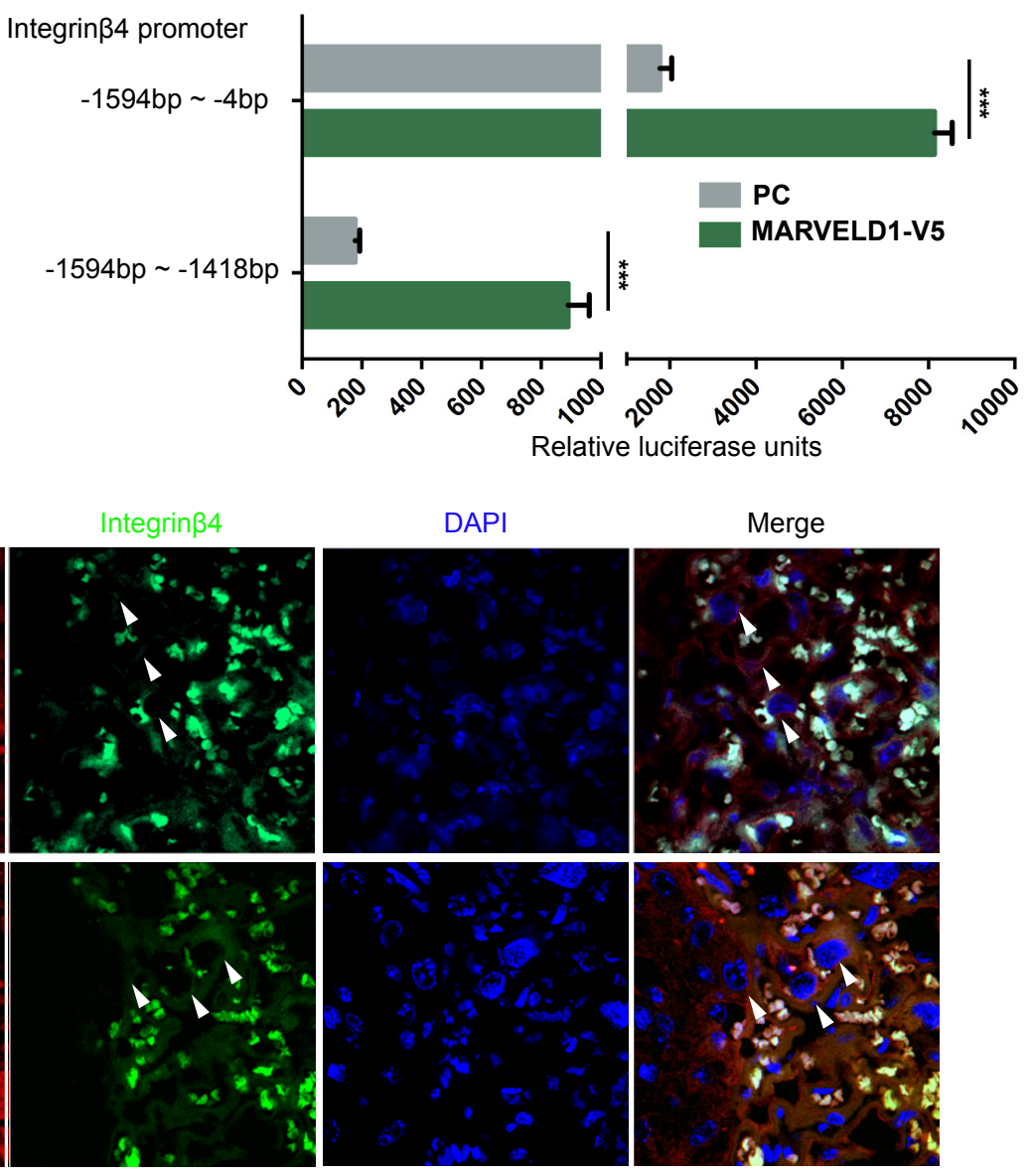

Relative luciferase units
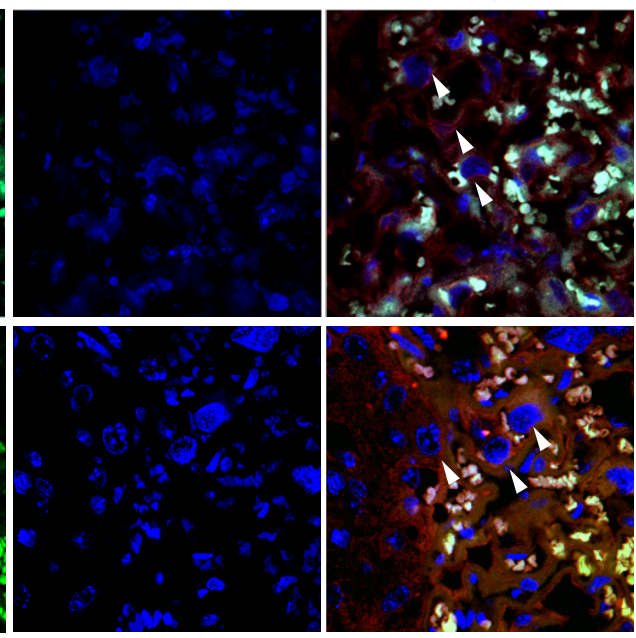

DAPI

Merge

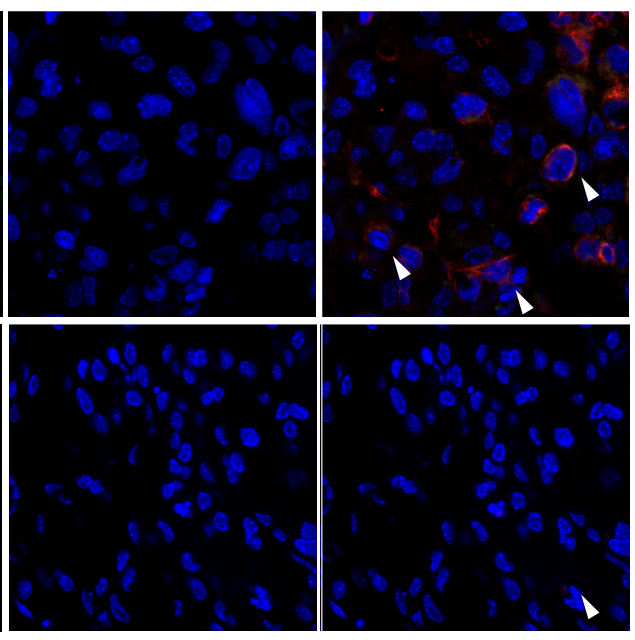

Figure 6 
A

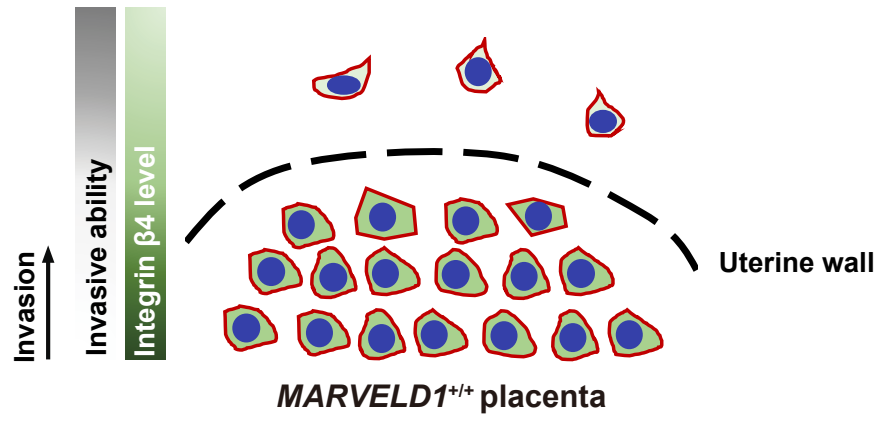

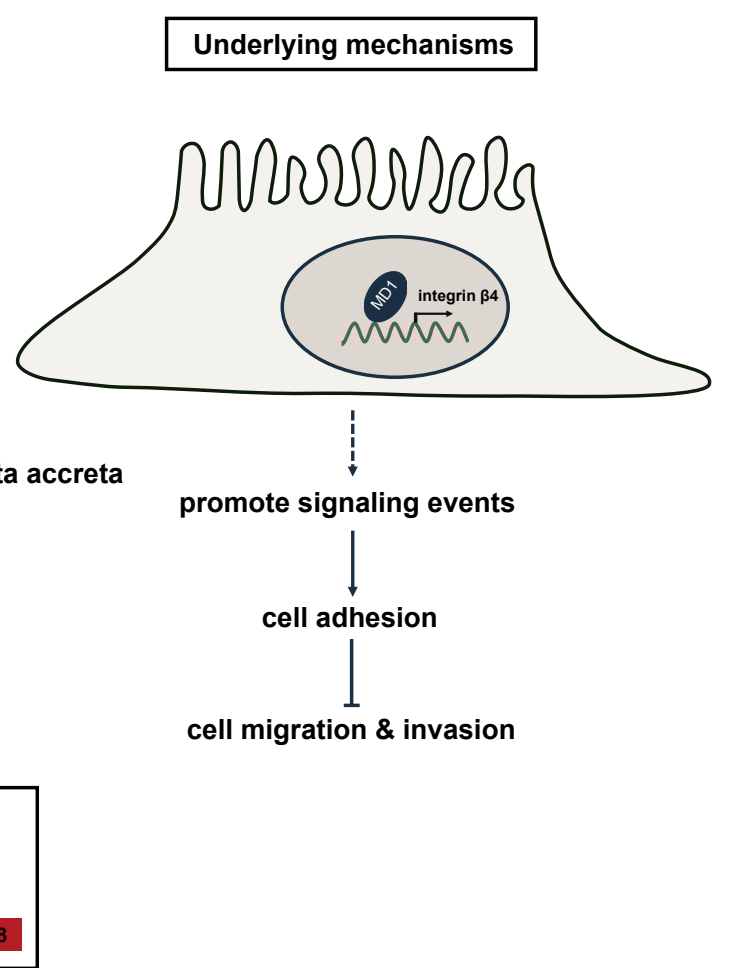

